\title{
Scanner Laser 3D de Baixo Custo
}

Cabral, R. J. R. O.

Escola Politécnica de Pernambuco

Universidade de Pernambuco

50.720-001 - Recife, Brasil

rayanmecatronics@gmail.com
Gómez-Malagón, Luiz A.

Escola Politécnica de Pernambuco

Universidade de Pernambuco

50.720-001 - Recife, Brasil

Resumo O processo de fabricação de peças ou componentes mecânicos segue uma metodologia bem definida e que faz parte do ciclo de vida do produto. Durante este ciclo, o desenho assistido por computador, também conhecido como CAD (Computer Aided Design) é a ferramenta empregada no início do projeto de qualquer produto. Com esta ferramenta é possível desenhar em três dimensões o objeto que queremos construir. Uma vantagem deste sistema é que o objeto virtual pode ser modificado facilmente e sem custos adicionais para o projeto. No entanto, o objeto virtual é construído dentro do programa CAD e não importado de um objeto real." Para solucionar este problema, foi construido um scanner para objetos tridimensionais que destina-se a importar o objeto real para o computador de maneira pratica e sem os altos custos de mercado que impedem a utilização deste tipo de dispositivo em larga escala.

\footnotetext{
Abstract The manufacturing process of parts or mechanical components follows a well-defined methodology which is part of the life cycle of the product. During this cycle, the computer aided design, also known as CAD (Computer Aided Design) is a tool used early in the design of any product. With this tool you can draw in three dimensions the object we want to build. An advantage of this system is that the virtual object can be modified easily and without additional cost to the project. However, the virtual object is built inside the CAD program and not imported from a real object. To solve this problem, one scanner for three-dimensional objects was built aiming to import the real object to the computer in a practical way and without the high costs of market that prevent the utilization of this type of device on a large scale.
} 


\section{Introdução}

No ciclo de vida de um produto, de início, o projetista deve identificar uma necessidade e seguidamente deverá propor algumas soluções ao problema. Estas soluções deverão ser analisadas individualmente, visando encontrar o ponto de equilíbrio entre o custo e o preenchimento dos requerimentos do projeto. Nesta fase, o designer emprega o uso de técnicas analíticas ou computacionais para escolher sua solução. Em alguns casos, é necessária a construção de um protótipo para validar a solução proposta e realizar as mudanças possíveis antes do projeto ser enviado para manufatura. Uma vez definida a solução, o projeto da peça é enviado para o setor de fabricação para depois a peça poder ser distribuída e comercializada. O cliente empregará a peça até que esta não preencha mais suas necessidades e seja descartada e, finalmente reciclada. Desta forma, o ciclo de vida do produto é fechado [1].

Durante este ciclo, ó desenho assistido por computador, também conhecido como CAD (Computer Aided Design) é a ferramenta empregada no início do projeto de qualquer produto. Com esta ferramenta é possível desenhar em três dimensões o objeto que queremos construir. Uma vantagem deste sistema é que o objeto virtual pode ser modificado facilmente e sem custos adicionais para o projeto. $\mathrm{O}$ objeto criado pode ser empregado para a análise de engenharia como, por exemplo, fazer a análise de tensões mecânicas, distribuição de temperatura, etc., usando a ferramenta computacional conhecida como CAE (Computer Aided Engineering). Uma vez que a peça atenda as exigências do projeto, o objeto virtual passa pela análise da manufatura no programa CAM (Computer Aided Manufacturing), onde é criado o código que será enviado para as maquinas $\mathrm{CNC}$ (Computer Numeric Control) para sua construção física [2].

Durante este processo, o objeto virtual é construído dentro do programa CAD e não importado de um objeto real. No entanto, durante o exercício da engenharia reversa, o objeto virtual é copiado do mundo real empregando um scanner, visando melhorar seu design e construir outra com as mesmas funcionalidades [2].

Uma tendência moderna na fabricação de componentes está no uso de tecnologias "on demand" ou técnicas de prototipagem rápida. Neste caso, a peça em formato digital é construída no mundo real usando uma impressora 3D. A grande vantagem deste modelo de manufatura é que o modelo 3D pode ser fabricado sem a necessidade de uso de programas de CAPP (Computer Aided Processes Planning) para fazer o planejamento da manufatura, isto quer dizer que não é necessário definir ferramentas de corte, analisar a s trajetórias da ferramenta previamente, visualizar colisões da ferramenta com a máquina, etc $[2,3]$.
Sendo assim, a junção das técnicas de "scanning" e prototipagem rápida, permitem ao projetista a construção de modelos que dificilmente poderiam ser construídos usando as técnicas convencionais de fabricação, como por exemplo, próteses [4].

\section{Técnicas Scanning}

Um SCANNER 3D é um dispositivo que analisa um objeto real para coletar dados sobre a sua forma e aparência. E com isto, os dados recolhidos podem então ser utilizados para construir modelos tridimensionais digitais. $\mathrm{Na}$ verdade, há uma longa fila de diferentes tecnologias que foram criadas para realizar essa tarefa. Isso não é tão surpreendente quando se considera o fato de que nenhuma tecnologia de digitalização $3 \mathrm{D}$ pode digitalizar toda a gama de diferentes objetos que nos rodeiam $[5,6]$.

Com o intuito de acomodar as diversas técnicas de digitalização, os métodos de scanning 3D podem ser classificados como de contato ou sem contato [7].

\subsection{Scanner 3D de contato}

Nesta técnica, a fim de compreender a geometria do objeto, um sensor de toque é guiado por um braço mecânico ou outro mecanismo coordenado por um sistema de medição automático ou assistido por um usuário (manual) que obedece o sistema de coordenadas cartesianas. A cada toque no objeto, um ponto terá a sua posição 3D registrada na memória e a qualidade do escaneamento resultante dependerá da quantidade de pontos registrados [6].

Entretanto, os scanners de contato são bastante lentos e geralmente não são usados para fazer modelos com um grande número de detalhes. Além disso, estes implicam em aparelhos volumosos possivelmente muito caros devido aos instrumentos necessários [6].

\subsection{Scanner 3D de contato}

Scanners sem contato incluem uma gama muito maior de tipos, porém, fornecem uma precisão menor do que sua versão de contato. Estes também costumam exigir mais envolvimento do usuário para configura-los corretamente. No entanto, scanners sem contato possuem taxas muito mais altas de varredura (varredura mais rápida). Estes podem ser mais facilmente modificados para digitalizar diferentes tamanhos de objeto. E, como o nome indica, scanners sem contato não tocam o objeto durante a digitalização. Essas propriedades os tornam muito populares. Além disso, alguns desses scanners podem realmente analisar dentro de certos objetos também. Por exemplo, o 
aparelho de ressonância magnética pode digitalizar dentro de um organismo vivo [6].

Os scanners sem contato utilizam algum tipo de radiação para fazer suas medições. Podem ser raios-x, lasers, infravermelho, campos magnéticos ou mesmo luz visível. As características específicas de cada radiação pedem técnicas específicas para processar os dados lidos e transformá-los em informações úteis. Um exemplo de scanner 3D sem contato é o MakerBot Digitizer [8].

A lógica geral de funcionamento de todo scanner 3D sem contato é a mesma. Este "conhece" as propriedades naturais da radiação que usa. Estas propriedades são levemente alteradas quando a radiação interage com qualquer objeto. Ela pode ser refletida, refratada ou sofrer algum outro tipo de interferência. Ao medir o que aconteceu com a radiação que interagiu com o objeto, ele pode inferir informações sobre o que causou estas alterações [8].

Quando o scanner usa a radiação ambiente (como a luz, por exemplo) ele é denominado scanner passivo. Por sua vez, os scanners ativos são aqueles que contém sua própria fonte de radiação [8].

\section{Objetivo}

Construir um Scanner laser de baixo custo.

\section{Materiais e Metodologias}

Provavelmente o tipo mais impressionante e bem conhecido dentro da categoria dos scanners sem contato é o scanner laser. Este é um dispositivo conta com um feixe de laser (linha de varredura), que é projetado sobre a superfície que vai ser copiada, e uma câmera de vídeo para coletar a imagem criada pelo laser sobre a peça. Vendo o objeto em um ângulo, a câmera irá revelar uma linha deformada que segue a curvatura da superfície do objeto. Usando o ângulo da câmera conhecido (ângulo entre a câmera e a linha de varredura), a posição $3 \mathrm{D}$ relativa de cada ponto da linha pode ser calculada. O objeto é então rodado de um incremento e o processo é repetido. Esta operação é realizada várias vezes modificando a posição do feixe laser sobre o objeto através de um atuador mecânico atuando na peça ou no feixe laser. Repetindo o processo em toda a superfície do objeto cria-se um conjunto completo em 3D do modelo de dados (mapeamento) do objeto. Este conjunto de dados contém pontos, cada um representando as posições 3D do objeto digitalizado. $\mathrm{O}$ objeto é construído, ligando esses pontos para formar uma circunferência, ou seja, representação do modelo 3D no computador [6].
Para construir tal dispositivo, inicialmente foi realizada uma revisão bibliográfica sobre as técnicas de digitalização de objetos 3D. Após a revisão foi proposto um sistema mecânico no qual foi modelado usando o software SolidWorks. O controle dos componentes do dispositivo tais como, laser, câmera e atuador mecânico foi realizado através da plataforma ARDUINO. Finalmente, foram testados alguns softwares disponíveis na internet e a escolha foi baseada no desempenho dos programas.

\section{Resultados}

O projeto para a construção do Scanner Laser 3D englobou um conjunto de processos nos quais foram realizados seguindo um plano de metas. Este teve sua pauta (Cronograma) dividida de maneira que primeiro houvesse a construção do Hardware (montagem do aparato mecânico e obtenção dos componentes eletrônicos) para depois ser realizada a manipulação dos Softwares com testes de sincronia entre os sistemas mecânico, eletrônico e computacional durante toda a execução do projeto.

\begin{tabular}{|c|c|c|c|c|c|c|c|c|c|c|c|c|c|}
\hline \multicolumn{14}{|l|}{ CRONOGRAMA } \\
\hline & Ano & \multicolumn{3}{|c|}{2013} & \multicolumn{9}{|c|}{2014} \\
\hline & Mes & 10 & 11 & 12 & 01 & 02 & 03 & 04 & 05 & 06 & 07 & 01 & 09 \\
\hline \multicolumn{14}{|l|}{ Revisäa biblingrifica } \\
\hline \multicolumn{14}{|l|}{ Modelaggenn mecảnica } \\
\hline \multicolumn{14}{|c|}{ Controle do mojor de passos } \\
\hline \multicolumn{14}{|c|}{ Centrole do feixe laser } \\
\hline \multicolumn{14}{|l|}{ Censtruçäo macanica } \\
\hline \multicolumn{14}{|l|}{ Anilisere de imagens } \\
\hline \multicolumn{14}{|l|}{ Teste do disposíno } \\
\hline Relatbinio de athindads & & & & & & & & & & & & & \\
\hline
\end{tabular}

Fig. 1. Plano de metas (Cronograma).

\subsection{Projeto Mecânico}

Antes da construção do protótipo, fez-se necessária uma abordagem gráfica virtual para facilitar possíveis modificações no esboço. Como o próprio nome indica, um Scanner Laser 3D de Baixo Custo exige que os materiais utilizados sejam baratos e de fácil acesso. Para isso, a modelagem do prototipo foi realizada através do SolidWorks e este foi construído considerando as propriedades do material utilizado, o custo e a sua disponibilidade no mercado. 


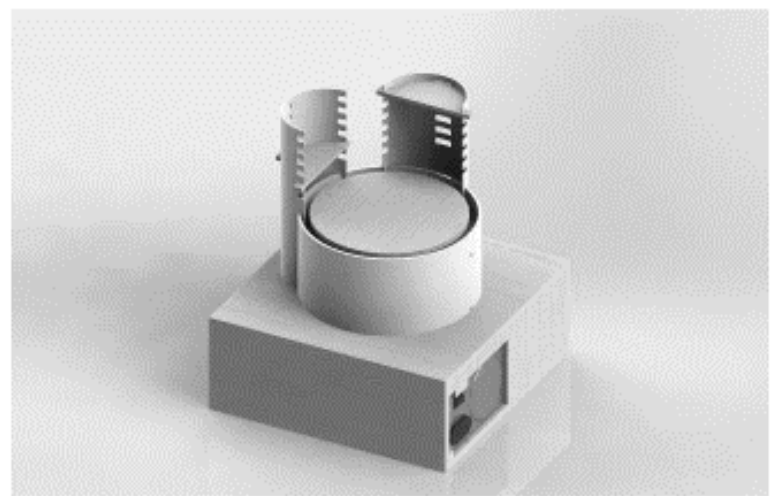

Fig. 2. Protótipo do Scanner (Solidworks).

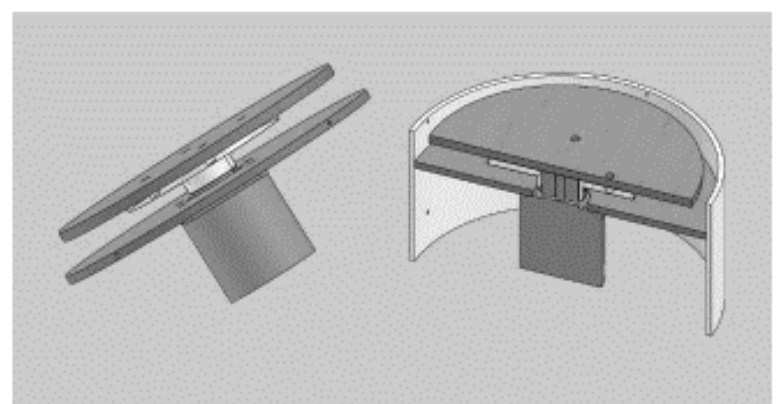

Fig. 3. Bloco superior (conjunto motor + prato).

Como indicado na Fig. 2, a modelagem conferiu ao projeto mecânico uma divisão em dois blocos. Pode-se verificar que o bloco superior, trata-se de um cano de PVC de $200 \mathrm{~mm}$ de diâmetro que comporta o sistema atuador. Este possui um motor de passo que é sustentado por um disco de madeira de mesmo raio que a borda interior do cano para que este fique justo, além de outro disco de madeira nomeado de "prato". O prato é conectado ao eixo do motor e possui a função de transmitir o movimento de rotação para o objeto de maneira mais aderente.

Por sua vez, o bloco inferior representado na Fig. 4 consiste de uma caixa de madeira montada para a acomodação dos circuitos eletrônicos, tais como uma fonte de alimentação e o Arduino. Enquanto elementos como Laser e a câmera tiveram de ser sustentados por uma "torre" que, como pode-se ver na Fig. 5, sofreu modificações durante a manufatura do projeto para melhor se adaptar com a angulação e a distância requerida. Os materiais utilizados para a sua construção foram suportes de prateleiras (cantoneiras) e conexões para tubos de PVC.

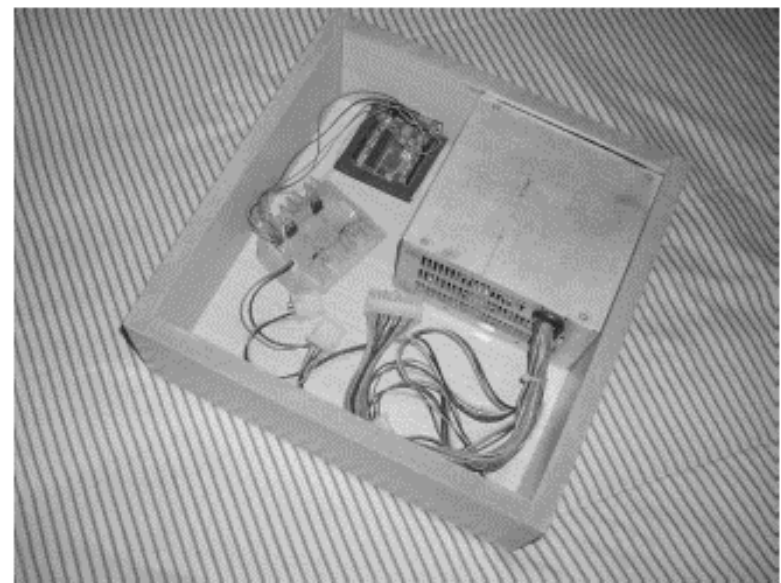

Fig. 4. Bloco inferior do Scanner 3D onde é possível visualizar o Arduino, a fonte e a placa confeccionada para auxiliar no controle do motor de passo.

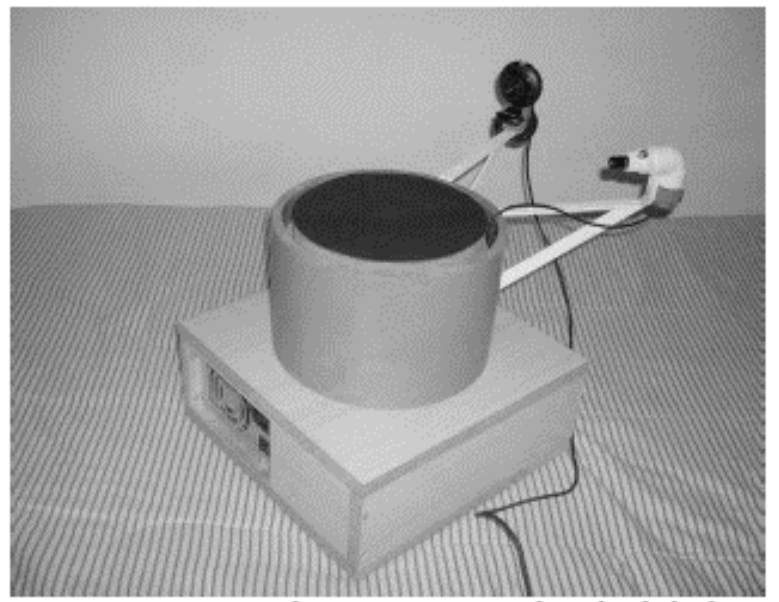

Fig. 5. Estrutura do Scanner completa incluindo as duas torres feitas com conexões de PVC e suportes de prateleira (cantoneiras).

\subsection{Projeto Eletrônico}

O Arduino foi fundamental para a conclusao do projeto. Este atua enviando pulsos através de suas portas digitais para cada uma das bobinas do motor de passo em uma sequência predefinida. Porém, o motor exige uma intensidade de corrente muito superior à que o Arduino pode oferecer. Para isto, uma placa foi confeccionada com o objetivo de chavear a passagem de corrente da fonte para cada fase do motor através dos pulsos do Arduino. Cada transistor contido na placa representa uma chave, onde o Arduino é ligado na base, a fonte no coletor e o motor no emissor. 


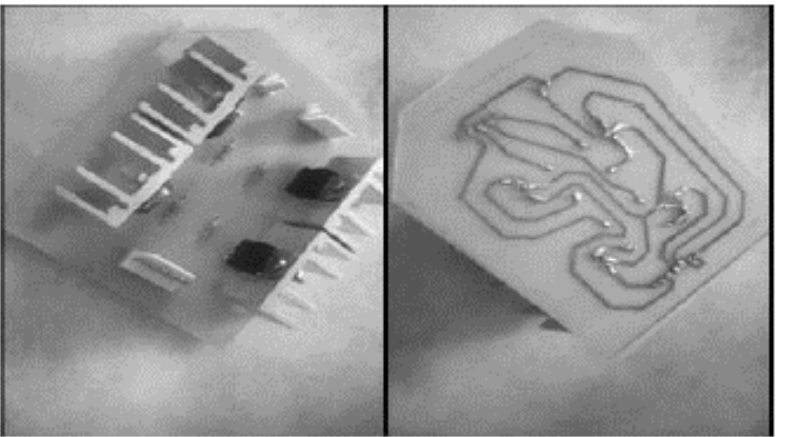

Fig. 6. Placa confeccionada para auxiliar no controle do motor de passo.

Outra instrução atribuída ao Arduino foi a simples função de ligar/desligar o Laser através de comando via porta serial. O laser é do tipo linear, como já dito na fundamentação teórica e, para a câmera, foi escolhida uma webcam devido ao custo reduzido.

Ao mesmo tempo, testes foram realizados a medida que cada meta era cumprida, criando um verdadeiro sistema mecatrônico onde cada elemento adicionado se tornava parte de uma rede de subsistemas administrados pelo usuário. Um exemplo prático é o fato de que quando a fonte tem seu interruptor acionado, a corrente flui através de seus fios até a placa confeccionada, encontrando as chaves transistorizadas em seu estado fechado. Com a ação do Arduino, pulsos elétricos sequenciais são enviados a base dos transistores alterando o estado das chaves para aberto, permitindo assim que a corrente volte a fluir e haja no motor de forma que cada uma de suas bobinas sejam acionadas e o prato inicie o processo de rotação segundo "passos". Como resultado disto, foram obtidas todas as ferramentas necessárias para o início da utilização do software de digitalização 3D.

\subsection{Software e Digitalização 3D}

A tecnologia de digitalização 3D existe há muitos anos. Apesar disso, este tipo de tecnologia não tem sido acessível para a maioria de nós. O nível de complexidade envolvido, o poder de computação disponível para processar e exibir os dados e, os elevados custos dos instrumentos necessários e desenvolvimento de produtos, são as três principais razões para isso [6].

Em virtude do que foi dito, optou-se por utilizar dois softwares devido a uma melhor compatibilidade apresentada com os sistemas operacionais mais comuns do mercado, sem exigir do computador grande capacidade de processamento ou qualquer custo adicional no orçamento do projeto, são eles "TriAngles 3D Circumference Scanner 2.0" e "TriAngles 3D Builder 2.0" (Copyright (C) 20062009 intricad).

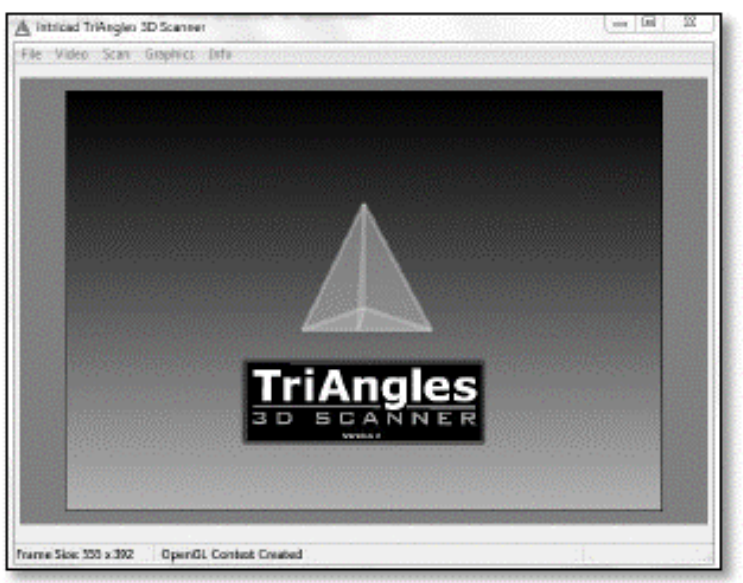

Fig. 7. Interface do software TriAngles 3D Scanner.

Para um melhor entendimento do processo de digitalização, o resultado final do escaneamento foi nomeado de "modelo" e este, por sua vez, é composto por dois elementos fundamentais para a sua criação. O primeiro, chamado "geometria", é o fator que define a forma final do modelo baseada na forma do objeto real e em sua interação com o Scanner. O outro elemento, denominado "textura", confere ao modelo as cores e a aparência superficial semelhantes as encontradas no objeto e, é através do TriAngles 3D Circumference Scanner 2.0 que pode-se obter ambos os elementos.

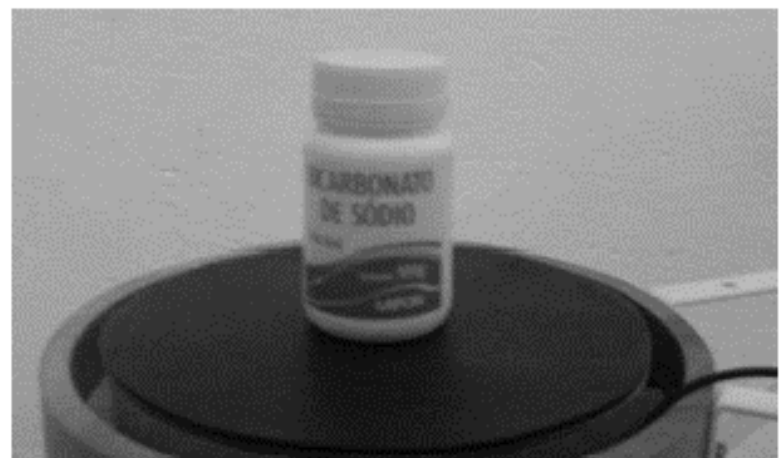

Fig. 8. Objeto escolhido para ser digitalizado (Pote de bicarbonato de sodio).

A escolha do objeto se baseiou em algumas limitacoes existentes em relacao as suas propriedades superficiais. $\mathrm{O}$ fato de este possuir uma superficie translucida, transparente ou conter grande capacidade de refletir, dificulta a acao de varredura, bem como a escolha de um objeto colorido implica em uma provavel alteracao na geometria do modelo. Isto acontece devido as propriedades oticas dos materias e das cores que confere a estes diferentes coeficientes de absorcao e reflexao. Por isto, o objeto escolhido foi fosco e bicromatico para facilitar a varredura e tambem, testar as capacidades do Scanner Laser 3D. 
O processo foi iniciado com o acionamento dos dispositivos contidos na estrutura do Scanner, isto é, a ligação da fonte na rede elétrica, além da conexão da webcam e do Arduino com o computador, esperando que o laser e o motor trabalhassem segundo as instruções enviadas pelo Arduino já explicadas neste artigo. Sobre o centro do prato, foi inserido o objeto a ser digitalizado e a webcam foi ligada por meio do TriAngles 3D Circumference Scanner 2.0. Com isto, a calibração da câmera foi realizada posicionando sua lente perpendicularmente ao prato em uma altura média do objeto de maneira que esta ficasse no centro da imagem, assim como o feixe laser. Após a calibração, foi importante manter o ambiente com baixa luminosidade para que, durante a varredura, o feixe laser tivesse um maior contraste em relação ao objeto, tornando possível a obtenção de melhores resultados. Além disso, foi essencial que o vídeo resultante capturasse os 360 graus da varredura para que todos os quadros (frames) necessários pudessem ser utilizados.

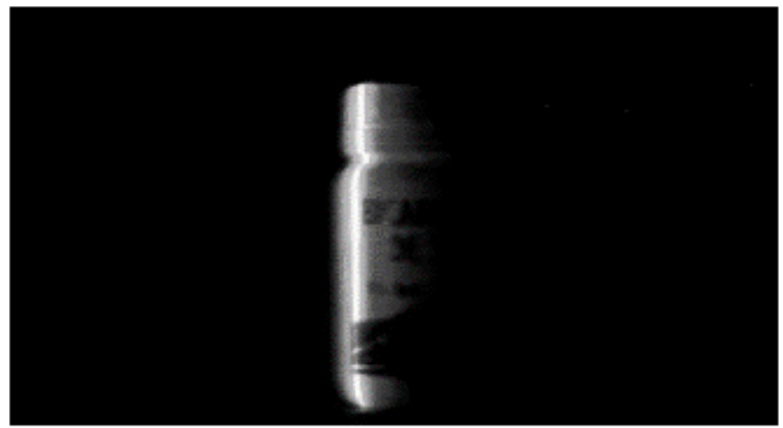

Fig. 9. Processo de Varredura.

Após isto, mais memória foi alocada e uma série de comandos tiveram de ser executados durante o processo. Um dos mais interessantes comandos do Triangles, o "Scan Line Isolation", permite que o molde da geometria seja ajustado de acordo com o nível de intensidade luminosa das zonas que o feixe percorreu. Após isto, o processo de compilação foi responsável pela construção da geometria.

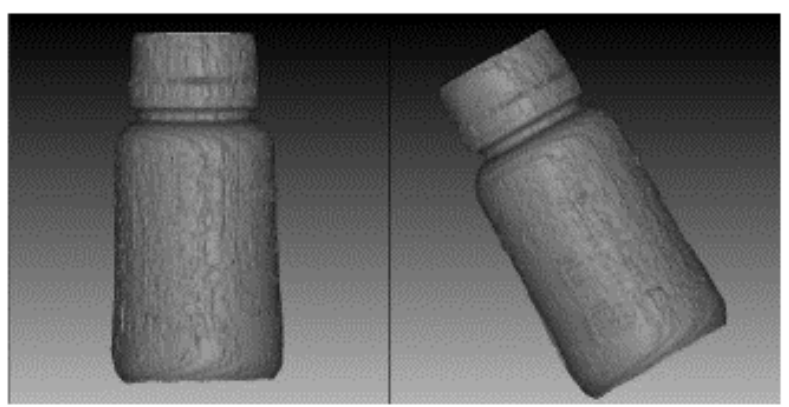

Fig. 10. Scan da geometria do objeto (Pote).

Para a obtenção da textura, foram seguidas as mesmas instruções do tópico anterior, exceto uma. Neste caso, um vídeo nítido e com uma boa qualidade implica em um melhor resultado. Então, através do processo de compilação, foi digitalizada a textura do objeto em 360 graus, finalizando a utilização do TriAngles 3D Circumference Scanner 2.0.

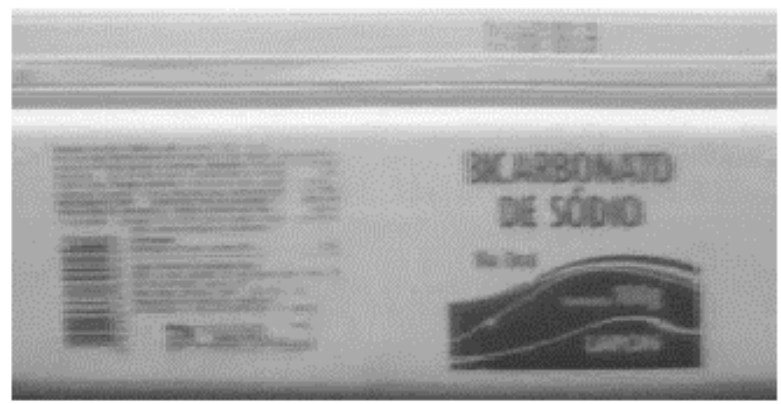

Fig. 11. Scan da textura do objeto.

Outras ferramentas que são essenciais em programas de digitalização 3D são os filtros. Estes são responsáveis pela diminuição das imperfeições da geometria gerada que comumente surgem durante o escaneamento. Utilizando o TriAngles 3D Builder 2.0 alguns filtros puderam ser conhecidos, testados e aplicados no decorrer do projeto:

- Suavização/Smooth: consiste na suavização da superfície da geometria do modelo com liberdade de aplicação tanto em pequenas áreas localizadas quanto em uma ampla gama de pontos.

- Deletar/Delete: apaga seções da superfície do modelo.

- Amolecimento/Soften: filtro muito similar a Suavização, no entanto, o seu índice de supressão de detalhes é mais elevado.

- Brilhar/Shine: aplicação de brilho.

- Polir/Add Gloss: aplicar aparência polida.

- Colorir/Color: Aplicação de cor.

Como mencionado, um modelo é uma composição de geometria e textura. Outra função do TriAngles 3D Builder 2.0 é unir estes dois elementos. Com isto, a textura foi aplicada sobre a geometria e a Fig. 12 revela o modelo concluído. 


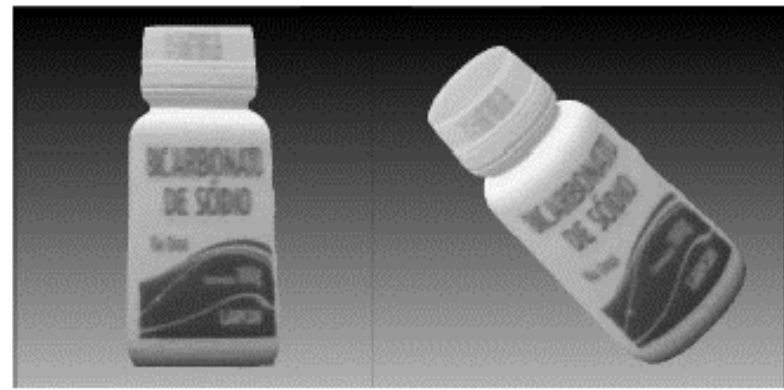

Fig. 12. Modelo - Objeto digitalizado (Pote). (Resultado Final)

Outro objeto, um Jarro, que apesar de ser colorido, tambem foi digitalizado com o intuito de comprovar a eficiencia do Scanner Laser 3D de Baixo Custo construido neste projeto.

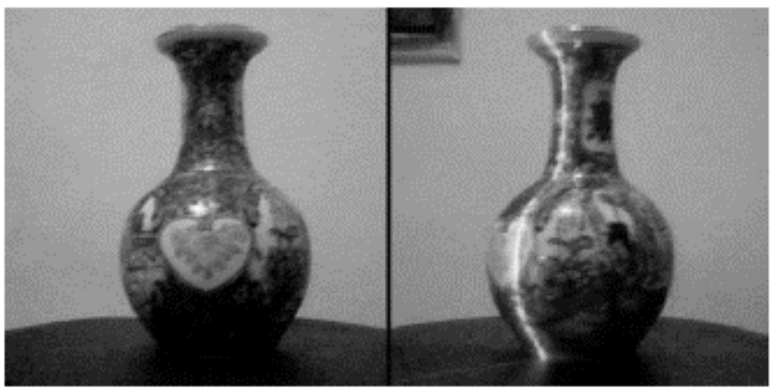

Fig. 13. Objeto escolhido para ser submetido a outra varredura e digitalizado.

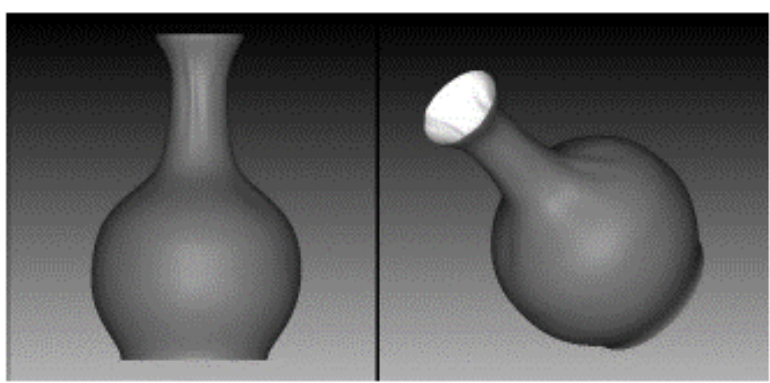

Fig. 14. Scan da geometria do objeto (Jarro).

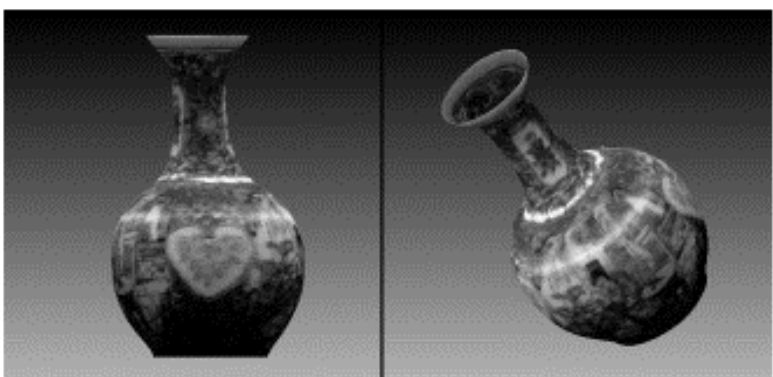

Fig. 15. Modelo - Objeto digitalizado (Jarro).

\section{Conclusão}

Algumas técnicas de prototipagem rápida estão cada vez mais incluídas em meio a sociedade em que vivemos. Um exemplo de dispositivo que aborda este tipo de técnica é a impressora 3D. Esta possui a função de imprimir quaisquer objetos já modelados em algum software de modelagem computacional. E, se usada em conjunto com um Scanner Laser 3D é possível a duplicação de objetos já existentes, bem como o aperfeiçoamento do seu design.

O Scanner Laser 3D é uma ferramenta inovadora que nos permite digitalizar objetos em três dimensões para um estudo mais aprofundado de sua geometria, visando uma análise mais confortável das suas dimensões e características. Além disso, é através deste dispositivo que é possível uma futura alteração do design de um produto já em fabricação, desconsiderando assim o árduo trabalho de remodelar o produto (se este o foi), partindo desde o seu início.

O Scanner Laser 3D construído neste projeto, foi modelado previamente de maneira a oferecer mais simplicidade para o usuário no sentido de facilitar a mobilidade e o uso do dispositivo. O Scanner consiste de apenas uma estrutura única, não sendo necessários vários aparatos e periféricos para o seu uso e, o que facilita no seu transporte é o fato de ser desmontável. Além disso, por apresentar uma estrutura composta por materiais de baixo custo de mercado como madeira e PVC por exemplo, este Scanner tornou-se um produto resultante muito mais barato, apresentando assim, bons motivos para atrair novos usuários.

A tecnologia envolvida no funcionamento do Scanner Laser 3D foi fundamentada em conceitos teóricos da eletrônica e da física, além de incluir a utilização do Laser, um dispositivo frequentemente empregado na área de fotônica. Vale relembrar que neste projeto, uma placa de circuitos foi produzida da a sua modelagem até a sua confecção.

O processo de digitalização foi realizado com o auxílio de softwares que tornaram possível a conclusão deste projeto. Os objetos apresentados foram submetidos ao processo de varredura e captação de sua geometria e textura de maneira cuidadosa, objetivando a obtenção do melhor modelo possível.

Em virtude de todos os fatos mencionados, o Scanner Laser 3D desenvolvido neste projeto apresenta grandes capacidades de digitalização, bem como um grande potencial de utilização devido a praticidade e o baixo custo que este possui advindo da necessidade de um produto mais barato no mercado. 


\section{Referências}

[1] M. P. Groover. Fundamentals of Modern Manufacturing: Materials, Processes and systems. Wiley (2010).

[2] M. P. Groover. Automation, Production Systems, and Computer-Integrated Manufacturing. Prentice Hall (2007).

[3] N. Volpato. Prototipagem Rápida. Ed. Blutcher (2007).

[4] L. Ciocca, R. Scotti, "CAD-CAM generated ear cast by means of a laser scanner and rapid prototyping machine," J. Prosthet. Dent. 92, 591-595 (2004).

[5] Wikipedia, 3D Scanner. Disponível em: <www.en.wiki pedia.org/wiki/3D_scanner>. Acesso em: 4 de setembro de 2014.

[6] Manual do programa de computador "TriAngles 3D Circumference Scanner, Version 2.0", Intri$\mathrm{cad}$

[7] IHS GlobalSpec, 3D Scanners Information. Disponível em: <www.globalspec.com/learnmore/manufacturing_pro cess_equipment/inspection_tools_instruments/3d_scanners>.

Acesso em: 6 de setembro de 2014.

[8] Manoel Lemos, Scanners 3D - descubra o que são e como funcionam. Disponível em: $<$ blog.fazedores.com>. Acesso em: 7 de setembro de 2014. 